\title{
Pressure Ulcer Prevention: Effectiveness of Heel Off-Loading Methodologies
}

\author{
Christopher C. Griffin1, Tammy Dean², Jonathan M. Cayce1, Mary Anne Modrcin² \\ ${ }^{1}$ DeRoyal Industries, Powell, TN, USA \\ ${ }^{2}$ Caylor School of Nursing, Lincoln Memorial University, Harrogate, TN, USA \\ Email: maryanne.modrcin@Imunet.edu
}

Received 18 September 2015; accepted 26 October 2015; published 29 October 2015

Copyright (C) 2015 by authors and Scientific Research Publishing Inc.

This work is licensed under the Creative Commons Attribution International License (CC BY). http://creativecommons.org/licenses/by/4.0/

(c) () Op Open Access

\begin{abstract}
Skin integrity breakdown and pressure ulcer formation on the heel are predictable and preventable healthcare occurrences. By minimizing surface contact pressures that can cause heel capillary occlusion, heel off-loading devices help mitigate the risk of decubitus ulceration. The purpose of this study is to investigate the efficacy of six off-loading methods commonly used in clinical settings to decrease heel contact pressure. Pressure mapping is used to evaluate contact pressure at the heel for underweight, normal weight, and overweight simulations. Based upon averages across four trials, it is shown that all devices reduce heel contact pressure when compared to no off-loading. The PRUventor ${ }^{\mathrm{TM}}$ boot exhibits significantly lower average pressure values than other off-loading strategies tested, suggesting that the PRUventor ${ }^{\text {TM }}$ is the most effective off-loading device. From the analysis of the experimental data and comparison to similar data for respective off-loading alternatives, it is concluded that the PRUventor ${ }^{\text {TM }}$ boot is an effective heel off-loading device for use in clinical settings.
\end{abstract}

\section{Keywords}

Heel Pressure Ulcer, Pressure Ulcer, Off-Loading Devices, Pruventor ${ }^{\mathrm{TM}}$ Boot, Pressure Mapping

\section{Introduction}

Pressure ulcer (PU) development is a serious concern in healthcare today. Patients who develop PUs are at greater risk for poor healthcare outcomes. Beginning in 2008, facilities no longer receive funds for services rendered for hospital-acquired PUs. Hospitals are focusing resources on prevention of PUs rather than treatment of PUs. Heel PU development is preventable when off-loading devices are utilized to decrease the amount of pressure exerted on the heel when a patient is on bedrest. 


\section{The Problem and Purpose of the Study}

As a patient becomes bed-ridden for an extended duration of time, significant and constant pressure to the heel can cause capillary occlusion, eventually leading to the development of tissue ischemia and a PU. The heel is at a higher risk of occlusion due to the thin protection layers between the skin surface and calcaneus prominence and decreased resting blood perfusion levels [1]. Additionally the calcaneus has a small surface area with a large weight bearing bony prominence, which results in higher than normal pressure. VanGilder, Amlung, Harrison, and Meyer [2] studied 450,000 patients and found heel ulcers accounted for $41 \%$ of all PUs. Pressure ulceration in medical facilities are classified as never events and the incidence of a PU is commonly used as an indicator for quality of care. In fact, the US government cites the development of PUs a "serious quality-of-care issue in all settings" [3].

The significance of patients acquiring PUs while hospitalized is multi-dimensional. Patients who develop PUs experience a unique set of consequences that includes an increased length of stay in the hospital, decreased quality of life, increased morbidity and mortality rates, and increased hospital costs. The purpose of this study was to investigate the efficacy of six off-loading methods commonly used in clinical settings to decrease heel contact pressure and thus the formation of heel PUs.

\section{Literature Review}

The root factor for the development of a PU is the lack of pressure relief on capillaries. "The capillary pressure generally is described as being approximately $32 \mathrm{mmHg}$ " [4]. If pressures higher than this are persistent, tissue ischemia and thus tissue necrosis results due to a lack of oxygen and nutrient supply to the tissues [4]-[6]. Torra I Bou et al. [6] concluded high pressure levels are often found in the heel area which predisposes patients to the development of a PU.

The incidence of PUs in general, and heel PUs specifically, is reported in the literature. There is a wide variation in the reported incidence of hospital acquired PUs due to various data collection methods and reporting practices. In 2009, Sanglard de Souza et al. [4] reported hospital units, excluding the Intensive Care Unit (ICU), had a PU occurrence rate of 3.5\% to 33\%. Lyder et al. [7] reported a PU occurrence rate of $15 \%$ but did not specify the hospital unit setting. The prevalence of PUs in patients with a fractured hip was found to be 21.3\% [8]. An older study by Nicosia, Giliatta, Woodbury, and Houghton [9] reported an annual rate of 1.6 million PUs in acute care settings in the United States. The incidence of PU development is higher for patients treated in the ICU. Cooper [5] reported a PU rate ranging from $10 \%$ to $41 \%$ and Sanglard de Souza et al. [4] reported a range of $19.2 \%$ to $44 \%$ of patients in the ICU develop a PU.

Specifically, PUs of the heel are the most common type of hospital-acquired PU, and the rate of heel PU is higher for patients treated in the ICU, [2] [10] [11]. Various studies have been conducted to pinpoint this number as well as assist in developing best practice guidelines for prevention of heel PUs. Campbell et al. [11] found the PU heel incidence rate to be $13.3 \%$ in hospitalized patients. Van Gilder et al. [2] estimated a $12.3 \%$ overall incidence of PU in hospitalized patients and of these pressure ulcers, $18.2 \%$ to $28.9 \%$ of overall and $41 \%$ of grade III and IV ulcers occur on the heel.

The cost of treating hospital-acquired PUs is tremendous. Since 2008, insurance companies no longer reimburse hospitals for services related to these types of PUs. One study found the annual cost to hospitals to treat PUs ranged from $\$ 2.2$ billion to $\$ 3.6$ billion [7]. In the same year, a study by Black [10] found there were 394,699 medical insurance claims filed in the United States related to hospital-acquired PUs, at a cost of $\$ 8730$ per case, for a total of $\$ 3.5$ billion. The cost to treat one stage III or stage IV PU ranges between $\$ 5000$ and $\$ 50,000$ [5]. It has been estimated the cost to provide general prevention of PU for hospitalized patients is $\$ 54.66$ per day [10].

A variety of risk factors related to the development of hospital-acquired PUs have been identified. These factors include age, ICU stays, lower extremity surgery, mechanical ventilation, decreased mobility, extended length of hospital stay, decreased level of consciousness, malnourishment, and a history of peripheral vascular disease[1] [5] [8] [9] [11]-[13].

The significance of PU development to the hospitalized patient is great. The literature mentions the more common results of PU development; increased cost, decreased quality of life, increased risk for below-the-knee amputation, limited rehabilitation, increased morbidity and mortality [11] [12]. Drake [14] reported more specific complications related to PU development as increased rates of infection, cellulitis, osteomyelitis, septice- 
mia, limb amputation, and death. McInerney [3] found a hospital-acquired PU is one of the top three in-hospital errors that lead to death. In fact, these patients were found to have a mortality risk 2 - 6 times greater than that of a patient with intact skin.

Prevention of PUs and specifically heel PUs should be a priority for the health care team. Frain [15] determined studies designed specifically for heel ulcer prevention are rare. There are some studies in the literature aimed at determining best practice guidelines for heel ulcer prevention. These studies typically look at utilizing some form of a heel device versus standard care in the prevention of heel PUs. Heel devices have shown to significantly reduce the incidence of heel ulcer development [1] [5] [12] [16]. Campbell et al. [11] concluded the use of a heel off-loading device is considered best practice for the prevention of heel PUs. This study also found the characteristics of the best PU prevention device included; it was cleanable, easy to use, cost effective, comfortable according to the patient, and available. Lyman [17] compared heel PU rates before and after the adoption of a heel off-loading device retrospectively in a 550 bed facility. The study followed an interrupted time series design consisting of a 6 month pre-intervention phase, a 3 month implementation phase, and a 6 month post intervention phase. In the pre-intervention phase, 39 pressure ulcers were observed compared to 2 pressure ulcers in the post-intervention phase demonstrating a 95\% reduction in pressure ulceration for the facility. The potential cost savings from this study was estimated to be $\$ 1,048,400$. Donnelly et al. [8] conducted a randomized controlled trial to determine the difference between heel PU development for patients who were treated with a complete heel off-loading device and those receiving standard care. Of the 119 patients in the control group, 31 patients (26\%) developed a PU with 8 of these PUs occurring on the heel. There were 120 patients who received a heel off-loading device and 8 of these patients (7\%) developed a PU with none being a heel PU.

\section{Purpose of the Study}

The studies included in the literature review demonstrate the effectiveness of heel off-loading devices for preventing heel PU formation; however, heel off-loading can be achieved through a number of different techniques. The question arises if one method or device is superior in terms of reducing the pressure on the heel when compared to other devices. To answer this question, we utilized pressure mapping technology to quantify and compare the contact pressures exhibited on the heel in a model leg by 6 methods off-loading methodologies currently employed in the clinic. Our null hypothesis was that there would be no difference in the amount of contact pressure exerted on the heel when various clinical methodologies and off-loading devices were compared.

\section{Method}

To test the effectiveness at relieving heel contact pressure, conditions were simulated for underweight, normal weight, and overweight body mass index conditions utilizing a hollow model leg. The model leg was loaded with various weight compositions to simulate the heel and leg weight exhibited by a 5 foot, 10 inch male as defined by the National Heart, Lung, and Blood Institute [18]. The model leg is a segment comprised of the shank and calf below the knee, ankle, and foot, made from a siliconized/rubberized plastic with foam filling in the foot (Figure 1(a)). Weight loading was done via combinations of hollow and solid aluminum shafts, a steel shaft, copper-plated steel BBs, and sand placed inside the manikin leg. Leg, shank, and foot weights were determined via Exercise Prescription body segment data [19]. Table 1 provides the leg weights for each simulated condition.
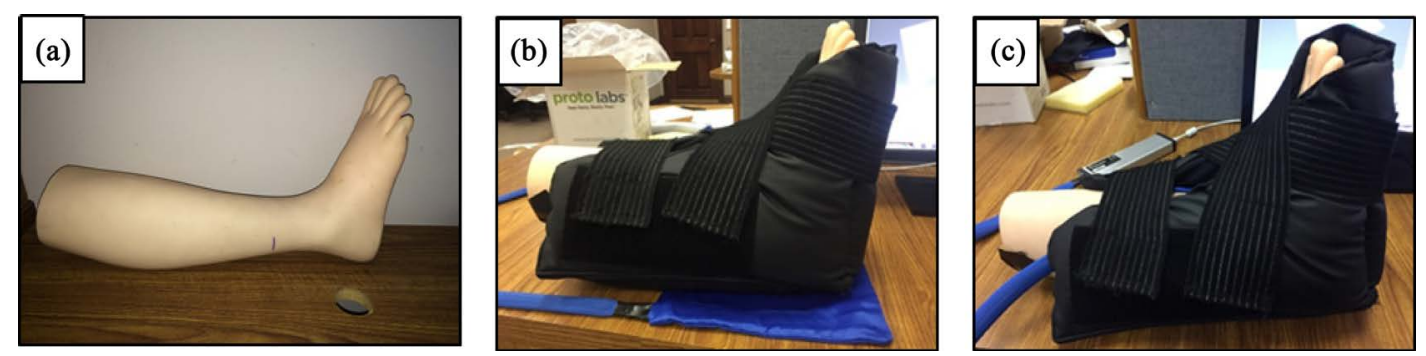

Figure 1. Orientation of control pressure mapping. (a) Manikin leg; (b) The leg is placed in the boot and set atop the Sensor Edge pressure mapping pad to gain an understanding of contact forces existing between surfaces; and (c) The sensor grid is wrapped around the heel and inserted into the boot to gain total heel contact measurements. 
Table 1. Simulated model leg loading weights for simulated body mass indexes.

\begin{tabular}{ccccc}
\hline & $\begin{array}{c}\text { Simulated Subject Weight } \\
\text { (Ibf) }\end{array}$ & Simulated BMI & $\begin{array}{c}\text { Simulated Target Leg Weight } \\
\text { (Ibf) }\end{array}$ & $\begin{array}{c}\text { Measured Manikin Leg Weight } \\
\text { (Ibf) }\end{array}$ \\
\hline Under Weight & 120 & 17.2 & 6.84 & 6.8 \\
Normal Weight & 170 & 24.4 & 9.69 & 9.15 \\
Over Weight & 220 & 31.6 & 12.54 & 12.25 \\
\hline
\end{tabular}

Six popular clinical methodologies and heel off-loading devices were evaluated with pressure mapping to determine the efficacy of each condition for off-loading the heel. The conditions tested in this study included:

- Control: No off-loading of the heel;

- Pillow Condition 1: Leg and foot resting on pillow;

- Pillow Condition 2: Leg resting on pillow with heel and foot extending beyond pillow end;

- Standard Foam Heel Pillow: Egg-shell foam designed to fit on the heel;

- Off-loading Device A: Heelift Glide ${ }^{\circledR}$ off-loading boot (DM Systems Inc.; Evanston, IL);

- Off-loading Device B: Prevalon ${ }^{\circledR}$ off-loading boot (Sage; Cary, IL);

- Off-loading Device C: PruventorTM off-loading boot (DeRoyal Industries; Powell, TN).

The initial pressure monitoring methods were based on the methods of Bush [20] where pressure mapping was used to demonstrate the off-loading efficacy of Off-loading Device B compared to no off-loading or offloading on a pillow (Pillow Condition 1). However, testing and review of the table-top sensor arrangement shown in Figure 1(b) revealed that this arrangement recorded the contact pressure between the surface and the off-loading device rather than total contact pressure to the heel (Figure 2). This led to a new method design which provided more accurate data regarding the pressure exerted on the heel by all contact points from the heel off-loading device. The sensor grid was wrapped around the heel then either placed inside or on top of the offloading device to obtain direct heel contact pressure readings (see Figure 1(c)). This arrangement allowed for a more accurate characterization of a device's heel off-loading efficacy. Pressure mapping of each off-loading methodology was measured by Sensor Edge's MeasureX software and flexible grip sensor pad (Parsippany, NJ). The MeasureX software was set to record 30 frames per second with a 100 moving average length value. The visualization palette range was set between 0 and 200. All data collected was recorded in units of mmHg.

For all conditions, the sensor grid was wrapped around the heel and ankle and secured with tape before insertion into/onto the off-loading device (Figure 1(c)). The leg was held in the air for several seconds before being set on the table during the initial data acquisition for calibration of the sensor grid. These initial data frame segments were subtracted out from the remaining data frames to remove the inherent contact forces that resulted from taping the sensor grid to the model leg and placing the model in or onto the off-loading device. Once the resting leg pressure readings stabilized, at least ten seconds of data was recorded for analysis. The investigator running the experiment located the center position of the heel and marked its location by indenting the pressure sensing mat. A masking grid that incorporated a 3 column by 4 row sensel array was centered on the heel location to determine heel contact pressures exerted on the heel during each condition. This grid size was chosen due to its geometric inclusion of heel contact area without significant effects of adjacent, zero-force sensel frames. The $3 \times 4$ grid creates a $19.35 \mathrm{~cm}^{2}$ sensel window, meeting the recommended $9-10 \mathrm{~cm}^{2}$ minimal measurement area for contact force determination on skin [21] [22]. The masking frame was used to only select data corresponding to heel to be exported from the MeasureX software.

Data analysis was performed in Microsoft ${ }^{\circledR}$ Excel $^{\circledR}$ (Microsoft Corporation) to determine the average contact force over the heel from each condition. This was accomplished by averaging all pressure values recorded within the masking frame over the ten second stabilization period for each trial. Four independent trials were conducted for each condition to generate a composite average for each BMI and an overall average for each condition obtained from the 12 total trials conducted for each offloading condition. Paired t-tests were utilized to identify significant differences between study conditions for the overall average heel contact force.

\section{Results}

The pressure mapping following Bush's [20] method illustrated in Figure 2 demonstrates that each of the offloading devices effectively lift the heel off the support surface and suggests the heel is completely floated; how- 
ever, this method is measuring only the contact forces between the boot and the support surface and not the contact forces between the heel and the off-loading device. Figure 3 displays pressure mapping with the sensor grid wrapped around the model foot and leg. This data demonstrates that each off-loading method reduces heel contact forces with the heel off-loading devices being more proficient at minimizing these forces. Further, stress concentrations are noticed in the areas adjacent to the heel prominence, particularly as the loading weight (BMI) increases. This is apparent in the calf and ankle regions, as seen throughout Off-loading Device A and occasionally in Pillow Condition 2, Off-loading Device B, and Off-loading Device C. The overweight case for Off-loading Device A in particular shows the correspondence of pressure application to the Achilles tendon. These pressures are caused by the dispersion of weight-induced contact forces across the leg, necessary to off-loading the heel, to the calf region where a natural cushion exists from muscle and fat tissue decreasing the likelihood of capillary occlusion.

Heel contact forces for each condition and trial were calculated by averaging 10 seconds of data from within the region of interest. Figure 4 visualizes the differences in off-loading efficacy between the conditions tested for each simulated BMI. Each off-loading condition was found to significantly reduce $(p<0.05$, paired t-test) heel contact forces compared to the control condition. Furthermore, Pillow Condition 2, the foam heel pillow, and each off-loading device significantly reduced the heel contact pressure when compared to Pillow Condition 1 . Interestingly, only Pillow Condition 2 and Off-loading Device C were found to significantly reduce the heel contact

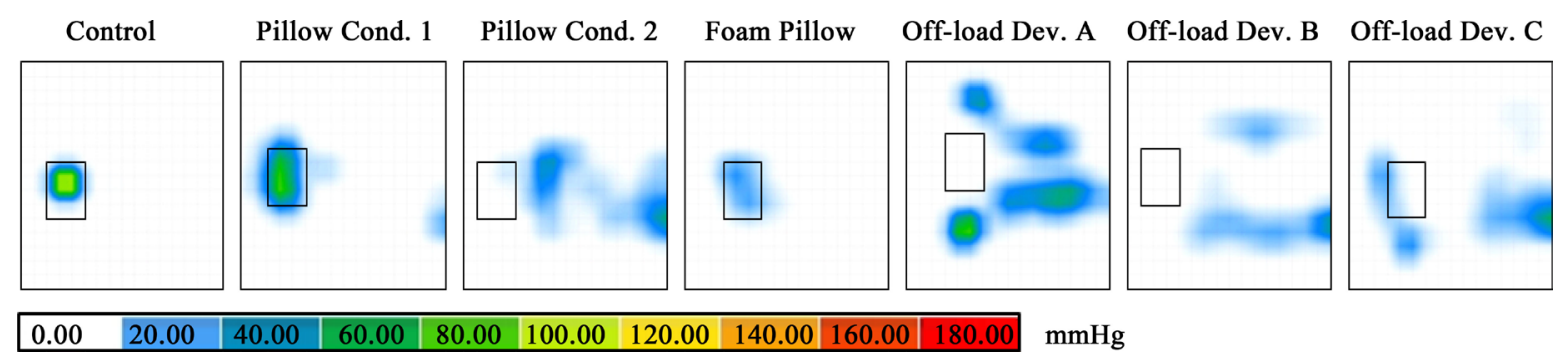

Figure 2. Pressure mapping utilizing method described by Bush 2009 [20]. Pressure maps displaying contact force distribution for each off-loading condition with pressure sensor placed between boot and table. The box indicates the location of the heel prominence. Additional external force was applied to show the orientation and location of the boot devices.

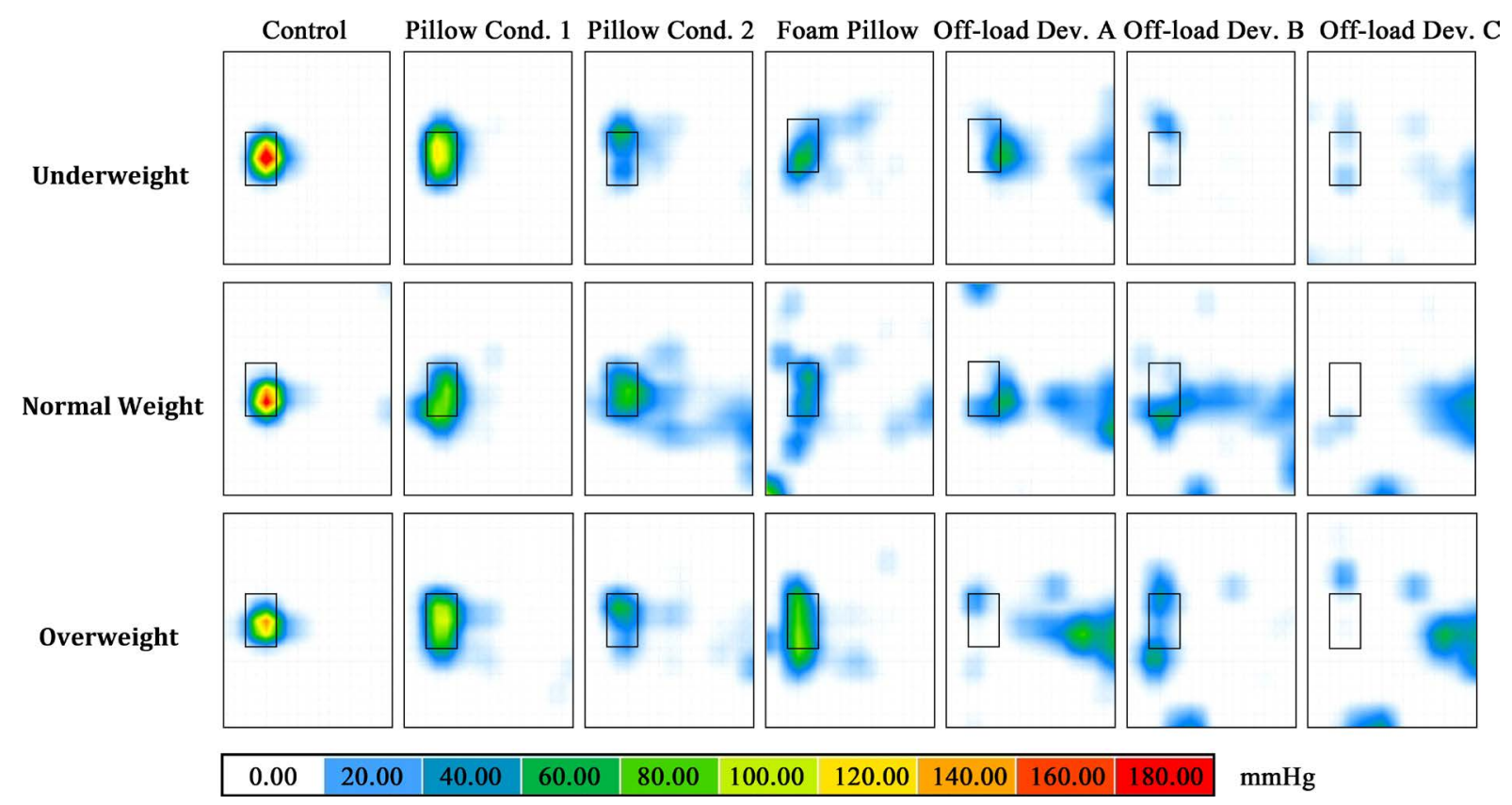

Figure 3. Pressure mapping visual comparison for pressure sensor attached to foot/leg complex. Pressure mapping visual comparison for pressure sensor attached to foot/leg complex. The rectangular box provides the location of the 3 column by 4 row sensel region of interest, as determined by heel location. 


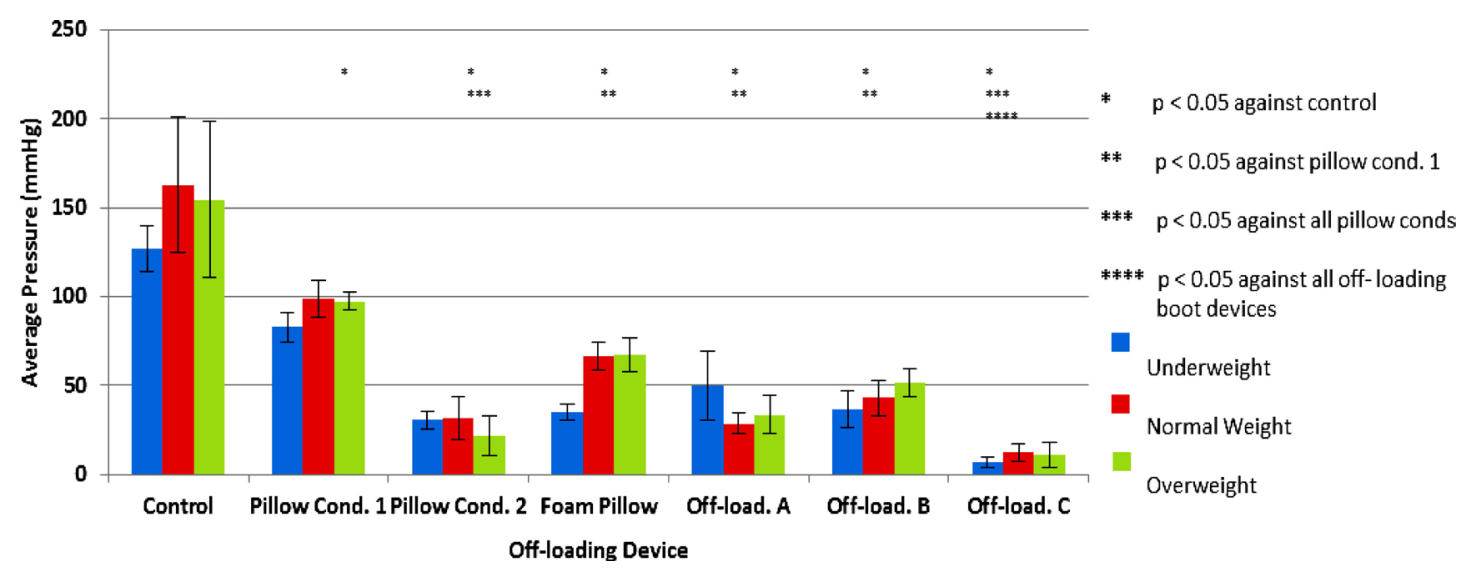

Figure 4. Data averages for each off-loading condition separated by loading amount. Standard error bars are displayed for each data set. Averages cover a twelve frame mask of heel forces averaged together over a ten second interval. Asterisks denote data significance as according to paired t-tests with a 5\% significance level.

pressure compared to the heel foam pillow. No significant differences were observed between Pillow Condition 2 compared to Off-loading Device A or B. Off-loading Device C was found to significantly reduce the heel contact pressure when compared to Pillow Condition 1, Off-loading Device A, and Off-loading Device B. These observations suggest that Off-loading Device C was most effective at reducing heel-contact forces.

\section{Discussion}

The results of this study demonstrate the ability of each condition to reduce heel contact pressure. Off-loading Device C (PRUventor ${ }^{\mathrm{TM}}$ ) proved to provide the greatest amount of heel off-loading across all three simulated BMIs (Table 1). Pillow Condition 2 exhibited similar performance, however, it should be noted that this condition is variable depending upon pillow compressibility, leg weight, and leg position. Donnelly et al. [8] showed that this method is not effective in clinical practice. Additionally, Pillow Condition 2 is particularly prone to external factors, such as movement and rotation of the foot, excessive force onto the ankle, and direct blanket pressure, which could significantly affect off-loading effectiveness. Paired t-tests analysis demonstrated Off-loading Device C significantly reduced heel contact pressure when compared with all other off-loading devices and methods ( $p$-value range: 8.92E-9 to 0.021). Both Off-loading Device A and Off-loading Device B, however, were found to be non-significant in relation to each other, the Foam Pillow, and Pillow Condition 2.

The capillary closing pressure is estimated to be at $32 \mathrm{mmHg}$ in healthy individuals and can be lower in compromised patients [4]; therefore, the average contact pressure on the heel should be less than $32 \mathrm{mmHg}$ for an off-loading device to be completely effective. Only Off-loading Device C averaged heel contact forces (10.00 mmHg overall), below $32 \mathrm{mmHg}$ for the three simulated BMIs (6.55 mmHg underweight, $12.31 \mathrm{mmHg}$ normal weight, and $11.14 \mathrm{mmHg}$ over weight). Off-loading Device A and B had an overall contact force average of $37.32 \mathrm{mmHg}$ and $43.75 \mathrm{mmHg}$ respectively. This observation would seem to indicate that these devices are not effective at preventing heel pressure ulcers; however clinical studies have demonstrated that these devices are effective at reducing the incidence of heel pressure ulceration in at risk patients [8] [17].

Considering the massive amount of evidence related to the cause, incidence, and significance of hospital-acquired PUs, it should be a priority for the healthcare team to focus on the prevention of PUs and specifically heel PUs. Pressure ulcer development in hospitalized patients is considered a never event but according to the literature, occurs in $33 \%$ of patients outside of the ICU and up to $44 \%$ of patients in the ICU. Awareness of PU development must be a priority when considering patient safety, health care outcomes, and risk management in the hospital setting. As patients are identified as high risk for the development of PUs, the health care team should implement guidelines directed at prevention of PUs. The literature clearly validates it is cost-effective to prevent PUs rather than treat them [5] [7] [10].

\section{Limitations of the Study}

The primary limitation of this bench side study is that it was performed on a model leg and not in human sub- 
jects. While the model leg was viewed as an adequate surrogate for the human leg, certain limitations and inherent error existed with the model. The primary limitation was the inability to load the leg with the same weight distribution in the leg from trial to trial due to movement of the weighting elements inside the leg when the model was moved. A slight change in the distribution of weighting elements likely contributed to variability seen in the data. Latent forces due to placing the sensor pad on the foot were removed by subtracting out initial readings with the leg and boot suspended in the air. While we view this correction as adequate, it is possible that all latent forces were not removed from the analysis or latent forces were applied during the calibration phase while the leg was held in the air. A second limitation lies in the characteristics of the off-loading device itself. While the pillow produced adequate results, different pillow compressibility or leg weights can cause the heel to come in contact with the supporting surface, negating the purpose of the off-loading device. Similarly, for devices with ankle contact, particularly Off-loading Device A, off-loading the heel creates a significant resultant pressure on the ankle portion that could produce skin abrasion and pressure sores. Also, the model did not allow for the study of leg mobility and exterior weighting that would be present in a clinical setting. Foot rotation, leg movement, and additional blankets and supports can all negatively affect off-loading device performance, particularly with pillow methods [8]. These limitations motivate the need for a clinical study utilizing humans of different BMI to confirm the pressure mapping results of this study.

\section{Conclusion}

Based upon the results of this study, it is concluded that heel off-loading devices and techniques effectively off-loads the heel for simulated underweight, normal weight, and overweight conditions. The pressure mapping of each condition shows dispersion of contact forces in boot devices to the calf and ankle, reducing risk of capillary occlusion. Further statistical analysis of the measured heel contact forces demonstrates that all devices reduce heel pressure, with Off-loading Device C (PRUventor ${ }^{\mathrm{TM}}$ ) providing the greatest degree of off-loading pressure relief compared to other conditions. These data trends support the general efficacy of the PRUventor ${ }^{\mathrm{TM}}$ boot under simulated conditions and motivate the need for further clinical research.

\section{Acknowledgements}

The authors would like to acknowledge DeRoyal Industries for sponsoring this study.

\section{Conflict of Interest}

CCG was recently employed and JMC is currently employed by DeRoyal Industries. TD and MAM have no conflicts of interest to disclose.

\section{References}

[1] Langemo, D. (2014) Heel Pressure Ulcers: 2014 International Pressure Ulcer Prevention \& Treatment Guidelines. 2014 National Pressure Ulcer Advisory Panel. www.npuap.org

[2] Van Gilder, C., Amlung, S., Harrison, P. and Meyer, S. (2009) Results of the 2008-2009 International Pressure Ulcer Prevalence Survey and a 3-Year, Acute Care, Unit Specific Analysis. Ostomy Wound Management, 55, 39-45.

[3] McInerney, J.A. (2008) Reducing Hospital-Acquired Pressure Ulcer Prevalence through a Focused Prevention Program. Advances in Skin \& Wound Care, 2, 75-78. http://dx.doi.org/10.1097/01.ASW.0000305410.58350.34

[4] de Souza, T.S., Danski, M.T.R., Johann, D.A., de Lazzari, L.S.M. and Mingorance, P. (2013) Prevention’s Pressure Ulcers Heel with Transparent Polyurethane Film. Acta Paul Enferm, 26, 345-352.

[5] Cooper, K.L. (2013) Evidence-Based Prevention of Pressure Ulcers in the Intensive Care Unit. Critical Care Nurse, 33, 57-66. http://dx.doi.org/10.4037/ccn2013985

[6] Torra I Bou, J.E., Lopez, J.R., Camanes, G., Narvaez, E.H., Blanco, J.B., Torralba, J.B., Soriano, J.V., et al. (2009) Preventing Pressure Ulcers on the Heel: A Canadian Cost Study. Dermatology Nursing, 21, 268-272.

[7] Lyder, C.H., Wang, Y., Metersky, M., Curry, M., Liliman, R., Verzier, N.H. and Hunt, D.R. (2012) Hospital-Acquired Pressure Ulcers. Journal of American Geriatric Society, 60, 1603-1608. http://dx.doi.org/10.1111/j.1532-5415.2012.04106.x

[8] Donnelly, J., Winder, J., Kernohan, W.G. and Stevenson, M. (2011) An RCT to Determine the Effect of a Heel Elevation Device in Pressure Ulcer Prevention Post-Hip Fracture. Journal of Wound Care, 20, 309-318. 
http://dx.doi.org/10.12968/jowc.2011.20.7.309

[9] Nicosia, G., Giliatta, A.E., Woodbury, M.G. and Houghton, P.E. (2007) The Effect of Pressure-Relieving Surfaces on the Prevention of Heel Ulcers in a Variety of Settings: A Meta-Analysis. International Wound Journal, 4, 197-207. http://dx.doi.org/10.1111/j.1742-481X.2007.00333.x

[10] Black, J. (2012) Preventing Pressure Ulcers Occurring on the Heel. Wounds International, 3, 58-61. www.woundsinternational.com.

[11] Campbell, K.E., Woodbury, M.G. and Houghton, P.E. (2010) Implementation of Best Practice in the Prevention of Heel Pressure Ulcers in the Acute Orthopedic Population. International Wound Journal, 7, 28-40. http://dx.doi.org/10.1111/j.1742-481X.2009.00650.x

[12] Tayyib, N., Coyer, F. and Lewis, P. (2015) A Two-Arm Cluster Randomized Control Trial to Determine the Effectiveness of a Pressure Ulcer Prevention Bundle for Critically Ill Patients. Journal of Nursing Scholarship, 47, $237-247$. http://dx.doi.org/10.1111/jnu.12136

[13] Thorpe, E. (2015) Pressure Ulcer Prevention in Intensive Care. Wounds UK, 11, 112-115.

[14] Drake, S. (2012) Implementing Evidence-Based Practice for Heel Pressure Ulcer Prevention in the Intensive Care Unit. Critical Care Nurse, 32, 45-46.

[15] Frain, R. (2008) Decreasing the Incidence of Heel Pressure Ulcers in Long-Term Care by Increasing Awareness: Results of a 1-Year Program. Ostomy Wound Management, 54, 62-67.

[16] Cadue, J.F., Karolewicz, S., Tardy, C., Barrault, C., Robert, R. and Pourrat, O. (2008) Prevention of Heel Pressure Sores with a Foam Body-Support Device. La Presse Médicale, 37, 30-36. http://dx.doi.org/10.1016/j.lpm.2007.07.009

[17] Lyman, V. (2009) Successful Heel Pressure Ulcer Prevention Program in a Long-Term Care Setting. Journal of Ostomy Continence Nursing, 36, 616-621. http://dx.doi.org/10.1097/WON.0b013e3181bd813e

[18] National Heart, Lung, and Blood Institute. Calculate Your Body Mass Index. http://www.nhlbi.nih.gov/health/educational/lose_wt/BMI/bmicalc.htm

[19] Exercise Prescription (2014) Body Segment Data. http://www.exrx.net/ Kinesiology/ Segments.html

[20] Bush, T.R. (2009) Pressure Mapping-Comparison of Unprotected Heels on a Hospital Bed vs. Heels Positioned in the Preval on Pressure Relieving Heel Protector. Sage Products. http://www.sageproducts.com/documents/pdf/education/case_studies/heel/21297.pdf

[21] Lung, C.W., Yang, T.D., Crane, B.A., Elliott, J., Dicianno, B. and Jan, Y.K. (2014) Investigation of Peak Pressure Index Parameters for People with Spinal Cord Injury Using Wheelchair Tilt-in-Space and Recline : Methodology and Preliminary Report. BioMed Research International, 2014, Article ID: 508583. http://dx.doi.org/10.1155/2014/508583

[22] Davis, K. and Sprigle, S. (2008) The Science of Interface Pressure Mapping-Updates for Clinical Application. http://hdl.handle.net/1853/36795 